\title{
Facial Expression Recognition Algorithm Based on PCNN
}

\author{
X. Jin, R.C. Nie, D.M. Zhou, J.F. Yu, K.J. He \\ Information College \\ Yunnan University \\ China
}

\begin{abstract}
A facial expression recognition method using the ignition position sequence (IPS) of pulse coupled neural network (PCNN) is proposed in this paper. First the method obtains normalized standard images by pre-processing the face images; and then uses PCNN model to extract the oscillation time sequences (OTS) of the face image, face identification is implemented by calculating the cosine distance of OTS and average OTS of all identities. Finally, face expression classification of testing face images is processed by using the IPS of PCNN. Experimental results in JAFFE expression database show that the method in this paper can efficiently distinguish the different expressions of the same identity, and achieve a higher recognition rate.
\end{abstract}

Keywords- facial expression recognition; pulse coupled neural network; oscillation time sequence

\section{INTRODUCTION}

With the rapid development of artificial intelligence and pattern recognition technology, biological information identification technology represented by face recognition [1], facial expression recognition [2], iris recognition [3], fingerprint recognition [4], which is gradually becoming a hot research field. As a very important part of man-machine interaction, facial expression recognition is drawing more and more attention from the researchers.

Generally, people's facial expressions have been divided into seven classes, namely, happy, sad, anger, surprise, fear, neutral and disgust. Its recognition goes through three processes: face detection, feature extraction and expression classification. Currently, the common facial expression recognition methods have, such as facial expression recognition using eigenspaces [5], face features and support vector [6], and template [7]. Although the first method can eliminate the invalid data to a large extent, but the result is unsatisfactory by limited features on behalf of face images. The second method based on face features and support vector emphasizes that the extracted features should preserve the facial information as much as possible. All the methods are not perfect because of the differentia of age, race, hair, and ornaments will be a greatly influence to the recognition results. In addition, human face is a flexible body, so it is difficult to establish a suitable ideal model for all facial expression recognition systems.

In view of the above analysis, we propose a facial expression recognition method based on the IPS of PCNN. In the method we first pre-process the input face images to get the standard face images. Then input the standard face image into PCNN model to discern the identity of the face image. Finally, the most important step is that we recognize facial expression by the IPS of PCNN. Experimental results in JAFFE expression database show that the proposed method was effective.

\section{FACIAL EXPRESSION RECOGNITION BASED ON PCNN}

\section{A. Face Image Pre-Treatment}

The interference of hair, neck and ornaments will disturb the recognition, so the face images need to be geometric normalization processing at first. In this method, we set the distance of two eyes as the reference of face image geometry normalization. Set $O$ as the midpoint of $E l$ (For the left eye), $\operatorname{Er}$ (For the right eye), $d$ is the connecting line between $E l$ and $E r$, rectangular face area is shown in Fig. 1. Finally, we get the standard image by scaling; the size is $150 \times 150$.

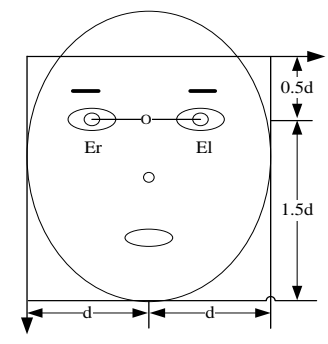

FIGURE I. PRE-TREATMENT RULE.

Intensity distribution of the image will also affect the ignition position of PCNN neurons, so before facial expression recognition is done, the grey scale of the image need to be normalized. We first find out the maximum brightness pixel of the image, denoted by $\operatorname{Imax}$, and $I^{\prime}=I / I \max$, where $I$ is pixel value of the original image, and $I$ ' is the adjusted pixel value of the image. After the geometric normalization and grey scale normalization are done, we will get the standard facial expression images with the size $150 \times 150$ and intensity uniformity.

\section{B. Identification Using PCNN}

Due to the different distribution of grey value, every image will have a unique OTS of PCNN. OTS is denoted by G[]. The OTS of PCNN has advantages of translational invariance, rotational invariance, scaling invariance, so it can effectively recognize identity of face image[8, 9,10$]$. In order to improve the accuracy of recognition, in this paper we use average OTS (denoted by $\overline{G[]}$ ) of different face image from the same 
identity [1]. We put $M$ face images from the same identity into PCNN model to get the average OTS, and then we will get the cosine distance between the OTS of the testing samples and the average OTS of every identity to achieve identification. OTS and average OTS see eqn. (1) and (2).

$$
\begin{aligned}
& G[]=\sum Y[n], \\
& \overline{G[]}=\frac{1}{M} \sum_{i=1}^{M} G_{i}[],
\end{aligned}
$$

where the binary image of PCNN's $n$th ignition is denoted by $Y[n], M$ is the sample number of the same identity.

\section{Facial Expression Feature Extracted by PCNN}

Facial expression, primarily caused by facial muscles, is expressed by eyes, nose, mouth, eyebrows, but there is no authorities' definition of facial expression. It is generally believed that people only have four facial expressions such as happiness, fear, anger, sadness, and other expressions are based on the refinement of these four expressions, in this paper we define facial expressions to have seven categories, namely, sadness, anger, happiness, surprise, fear, neutral and disgust.

The OTS of PCNN has the advantage of less data and good robustness, and it has showed superior performance in face recognition [10]. The OTS of the same identity is very similar, but it may also be close if the different identities had a similar face. So the OTS cannot adequately reflect the distribution of grey value and the minutiae of the face images. And the output binary images of PCNN neuron in every times of iteration are the neuronal ignition position information, see Fig. 2 (b), (c), (d) shown the 5th, 7th, 9th ignition position of the face image Fig. 2 (a). The position images of every iteration ignition not only reflect the detail features of the face from the different point of view, but also can reflect the nuance of different expressions.

We can draw a conclusion that the date of each ignition image is too much to deal with, and it is also not conducive to be used for facial expression recognition, so we need to compress the data. Firstly, $N$ iterations ignition position information is superimposed integrated ignition position information denoted by $I(x, y)$, see eqn. (3). Then, projecting the face image to horizontal direction and vertical direction; we will get the feature vector $P H$ and $P V$, see eqn. (4) and (5). Finally, add feature vector $P H$ to feature vector $P V$ to get vector $V H$, see eqn. (6), $V H$ is the vector of the facial expression.

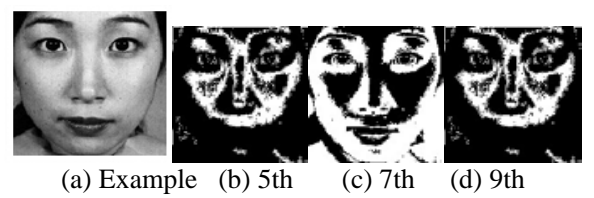

FIGURE II. EXAMPLE FACE IMAGE AND ITS PCNN ITERATION IGNITION POSITION.

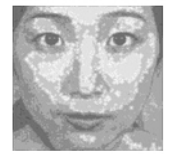

(a) IPS

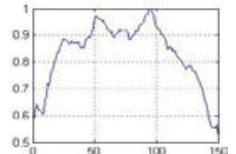

(b) $V H$
FIGURE III. IPS AND FEATURE VECTOR VH

$$
\begin{gathered}
I(x, y)=\sum_{N=1}^{N} Y_{i j}[n], \\
P H(y)=\sum_{x=1}^{n} I(x, y), \\
P V(x)=\sum_{y=1}^{m} I(x, y), \\
V H=\frac{1}{2}\left(P H+P V^{T}\right) .
\end{gathered}
$$

The face image in Fig. 2(a) is input to PCNN model, and then the 20 times iteration ignition position sequence (IPS) are superimposed to get Fig. 3(a), which reflects the position information of PCNN iteration ignition. Fig. 3(b) is characteristic curve $\mathrm{VH}$. The distribution information in the horizontal and vertical can be integrated to reflect the ignition of each pixel in the face image. It can be seen, PCNN IPS cannot only reduce the amount of data, but also reflect the position distribution information of neuronal firing repeatedly, and the IPS of different expression is very different.

\section{Expression Classification Criteria}

Since the cosine distance on discrete data has good robustness, so we choose cosine distance to discriminate different expressions. In the experimental process,

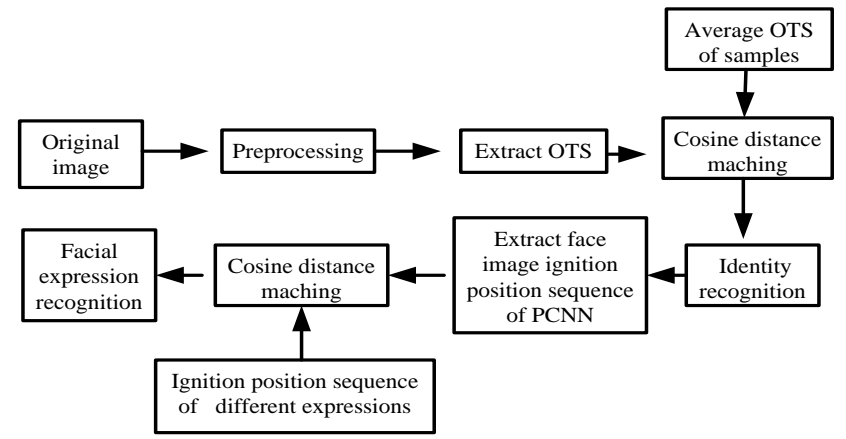

FIGURE IV.EXPRESSION RECOGNITION ALGORITHM PROCESSES.

the IPS of the testing face images as its feature vector, denoted by $V H_{\text {test }}[]$. Seven kinds of expression vector group of the same identity denoted by $\mathrm{VH}_{7}[]$. In the testing process, first recognize the identity, and then calculate the cosine distance between $V H_{\text {test }}[]$ and $V H_{7}[]$, see eqn. (7). The sample facial expression of the maximum cosine distance is corresponding to the testing facial expression. The process of facial expression recognition algorithm is shown in Fig. 4.

$$
\operatorname{Cosin} e\left[V H_{\text {Test }}, V H_{7}\right]=\frac{V H_{\text {Test }}[] \cdot V H_{7}[]}{\left\|V H_{\text {Test }}[]\right\| \cdot\left\|V H_{7}[]\right\|}
$$

\section{COMPUTER SIMULATION RESULTS AND ANALYSIS}

The experiment use a static JAFFE expression database which consists of 213 face images of 10 Japanese women, each has facial expression with anger, disgust, fear, happiness, neutral, sadness and surprise, and there are 2-4 samples for each person. The PCNN model used in the experiment is the original model, which model parameters are $\quad \beta=0.1, \alpha_{L}=1, \alpha_{E}=1, \alpha_{F}=0.1, \quad V_{L}=0.1, V_{F}=0.5, V_{E}=20$, $W=M=[0.5,1,0.5 ; 1,0,1 ; 0.5,1,0.5]$. 


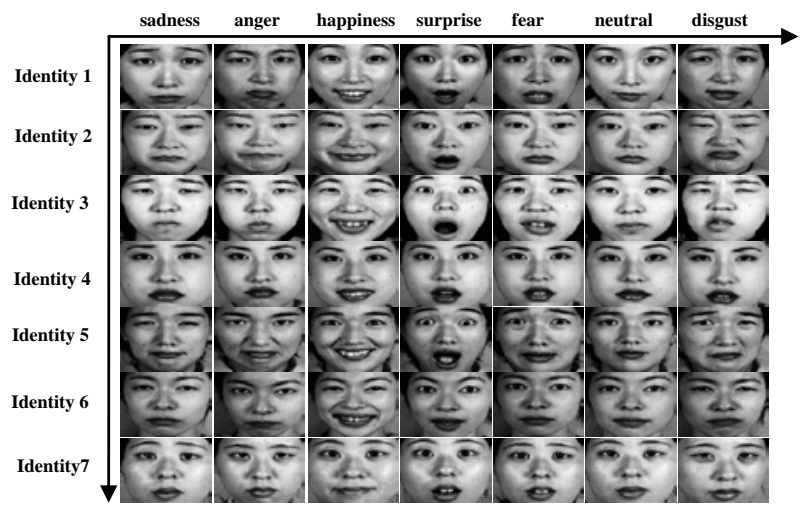

FIGURE V. IDENTITY/EXPRESSION SCHEMATIC.

Fig. 5 shows the schematic diagram of 7 kinds of identities and 7 corresponding expressions in JAFF expression database. In the experiment, face identification first along the longitudinal axis, and then facial expression classification along the horizontal axis.

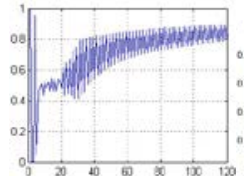

(a) Sadness

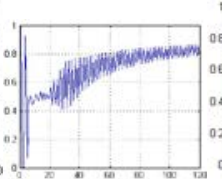

(b)Anger

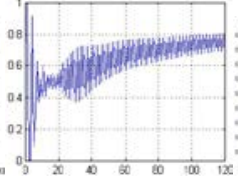

(c) Happiness

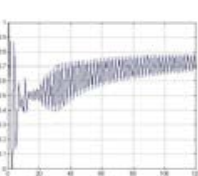

(d) Neutral

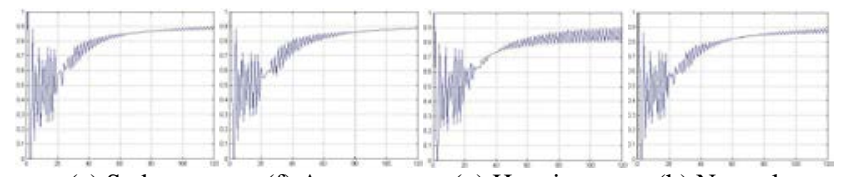

(e) Sadness (g) Happiness

\section{FIGURE VI. OTS OF EXPRESSION OF IDENTITY 1 AND 2 IN FIG5.}

Fig. 6 is the OTS of four corresponding expressions of identity 1(a, b, c, d) and identity 2(e, f, g, h) in Fig. 5 respectively. It can be seen from the figures, the expressions from the same face are apparently different, but the corresponding OTS is relatively similar, the OTS from different face varies widely.

TABLE I. IDENTITY RECOGNITION RESULTS(RR: RECOGNITION RATE).

\begin{tabular}{|l|l|l|l|l|l|l|l|l|l|l|}
\hline $\begin{array}{l}\text { Identit } \\
\text { y }\end{array}$ & 1 & 2 & 3 & 4 & 5 & 6 & 7 & 8 & 9 & 10 \\
\hline $\begin{array}{l}\text { Sampl } \\
\text { e }\end{array}$ & 6 & 6 & 6 & 6 & 6 & 6 & 6 & 6 & 6 & 6 \\
\hline $\begin{array}{l}\text { Test } \\
\text { Set }\end{array}$ & 17 & 16 & 16 & 14 & 15 & 15 & 14 & 14 & 15 & 16 \\
\hline $\begin{array}{l}\text { Errors } \\
\text { Total }\end{array}$ & 4 & 3 & 0 & 3 & 2 & 3 & 3 & 1 & 2 & 2 \\
\hline $\begin{array}{l}\text { RR(\% } \\
\text { ) }\end{array}$ & $\begin{array}{l}76 \\
.5\end{array}$ & $\begin{array}{l}81 \\
.3\end{array}$ & $\begin{array}{l}10 \\
0\end{array}$ & $\begin{array}{l}78 \\
.6\end{array}$ & $\begin{array}{l}86 \\
.7\end{array}$ & $\begin{array}{l}80 \\
.0\end{array}$ & $\begin{array}{l}78 \\
.7\end{array}$ & 92.9 & $\begin{array}{l}86 \\
.7\end{array}$ & $\begin{array}{l}87 . \\
5\end{array}$ \\
\hline
\end{tabular}

Table 1 shows the 10 identities recognition results using OTS in JAFFE expression database. It can be seen from Table 1 , the method can effectively recognize identifies, and the average correct recognition rate is $85.53 \%$. The errors in the experiment is due to the diversity in the facial expression database, plus some people's faces are similar, which is shown by identity 2 and identity 5 in Fig. 5 , so it will lead to some different faces have the similar OTS to bring about the misjudgement.

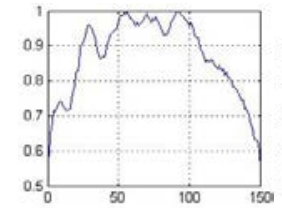

(a) Sadness

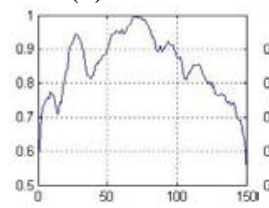

(c) Happiness

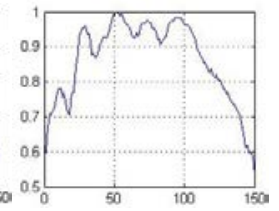

(b) Anger

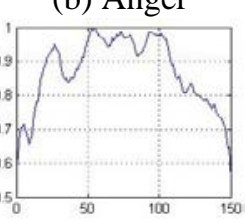

(d) Neutral
FIGURE VII. IPS OF EXPRESSION OF IDENTITY 2

Fig. 7 shows the IPS of 4 kinds of expression of identity 2 in Fig. 5, it can be seen from the Fig. 8, the IPS of different expression from the same identity have large difference, in addition to this the IPS of the same expression from the same identity have same similar curve. So we can realize facial expression recognition by IPS. Table 2 shows the experimental results in JAFFE expression database using the IPS of PCNN. In Table 2, 1/2 denotes correct number/sample number. It can be seen, the method can effectively distinguish the different expression, and in particular it shows a higher recognition rate and robustness in the case of known identity.

TABLE II. EXPRESSION RECOGNITION RESULTS(RR: RECOGNITION RATE).

\begin{tabular}{|l|l|l|l|l|l|l|l|l|l|l|l|}
\hline & 1 & 2 & 3 & 4 & 5 & 6 & 7 & 8 & 9 & 10 & $\mathrm{RR}(\%)$ \\
\hline Sadness & $1 / 2$ & $2 / 2$ & $3 / 3$ & $1 / 2$ & $2 / 2$ & $2 / 2$ & $2 / 2$ & $1 / 1$ & $1 / 2$ & $2 / 2$ & 90.0 \\
\hline Anger & $2 / 2$ & $2 / 2$ & $2 / 2$ & $2 / 2$ & $2 / 2$ & $1 / 2$ & $0 / 2$ & $1 / 2$ & $1 / 2$ & $2 / 2$ & 75.0 \\
\hline Happiness & $3 / 3$ & $2 / 2$ & $3 / 3$ & $1 / 1$ & $2 / 2$ & $3 / 3$ & $2 / 2$ & $2 / 2$ & $2 / 2$ & $2 / 2$ & 100.0 \\
\hline Surprise & $2 / 2$ & $2 / 2$ & $2 / 2$ & $2 / 3$ & $2 / 2$ & $1 / 1$ & $2 / 2$ & $2 / 2$ & $2 / 2$ & $2 / 2$ & 95.0 \\
\hline Fear & $2 / 3$ & $2 / 2$ & $2 / 2$ & $2 / 2$ & $2 / 2$ & $2 / 2$ & $1 / 2$ & $2 / 2$ & $2 / 2$ & $1 / 2$ & 85.71 \\
\hline Neutral & $2 / 2$ & $1 / 2$ & $1 / 2$ & $2 / 2$ & $2 / 2$ & $1 / 2$ & $2 / 2$ & $2 / 2$ & $2 / 2$ & $2 / 2$ & 85.0 \\
\hline Disgust & $2 / 2$ & $3 / 3$ & $1 / 1$ & $1 / 2$ & $2 / 2$ & $2 / 2$ & $1 / 1$ & $2 / 2$ & $1 / 2$ & $2 / 2$ & 89.47 \\
\hline
\end{tabular}

\section{CONCLUSIONS}

In this paper, a facial expression recognition method based on the IPS of PCNN was proposed. Firstly, using the average
OTS of PCNN to recognize identity, because of the OTS has the advantage of translational invariance, rotational invariance, scaling invariance, and then using the IPS of PCNN to carry 
out facial expression recognition. The experimental results show the method in this paper can effectively implement face identification and facial expression classification with good robustness and applicability.

\section{ACKNOWLEDGEMENTS}

Our work is supported by the National Natural Science Foundation of China (No.61365001, No.61463052), Natural Science Foundation of Yunnan Province (No.2012FD003).

\section{REFERENCES}

[1] R.C. Nie, S.W. Yao, D.M. Zhou, Face recognition using Simplified Pulse Coupled Neural Network. Computer Science, 41(2): pp.297-301, 2014.

[2] Y. Luo, C.M. Wu, Y. Zhang, Facial expression recognition based on principal component analysis and support vector machine applied in intelligent wheelchair. Application Research of Computers, 29(8): pp.3166-3168, 2012.

[3] X. Jin, R.C. Nie, D.M. Zhou, An improved iris recognition algorithm based on PCNN. Computer Science, 41(11A): pp.110-115, 2014.

[4] C. Moujahdi, G. Bebis, S. Ghouzali, et al. Fingerprint shell: Secure representation of fingerprint template. Pattern Recognition Letters, 45: pp.189-196, 2014.

[5] D. Chakrabarti, D. Dutta, Facial Expression Recognition Using Eigenspaces. Procedia Technologys, 10: pp.755-761, 2013.

[6] L.Y.Dong, L. Liu, X.F. Wang, A Face Expression Recognition Method Based on Face Features and Support Vector Machine. Journal of Hefei University (Natural Sciences), 19(1): pp.24-27, 2009.

[7] C.C. Lai, C.H. Ko, Facial expression recognition based on two-stage features extraction. Optik, 125: pp.6678-6680, 2005.

[8] J.L. Johson, D. Ritter, Observation of periodic wanes in a pulse-coupled neural network. Optics Letters, 18(15): pp.1253-1255, 1993.

[9] M.M. Subashini, S.K. Sahoo, Pulse coupled neural networks and its applications. Expert Systems with Applications, 41: pp.3965-3974, 2014.

[10] L. Wang, N. Wang, L. Shen, et al, Face recognition based on semisupervised manifold learning. Computer Engineering And Applications, 47(17): pp.192-195, 2011. 